\title{
Electromagnetism as a world view: implications for the teaching of energy
}

\author{
Fabiana Botelho Kneubil ${ }^{* 1,2}$, Ricardo Karam², Ivã Gurgel ${ }^{3}$, Manoel Roberto Robilotta ${ }^{3}$ \\ ${ }^{1}$ Department of Physics, Federal University of Santa Catarina, Brazil \\ ${ }^{2}$ Department of Science Education, University of Copenhagen, Denmark \\ ${ }^{3}$ Institute of Physics, University of São Paulo, Brazil
}

Received on April 10, 2017. Accepted on May 14, 2017.

\begin{abstract}
There are considerable differences in the ways mechanics and electromagnetism conceptualize energy. In the former, energy is defined in terms of force and work in a process-like reasoning that remits us to the "past" of a given body or system. Moreover, in the mechanical framework energy is ascribed to matter, i.e., one speaks of the energy of a system of bodies. The electromagnetic picture is quite different. There, energy assumes a form localized directly in the fields, i.e., if a region in space contains $\vec{E}$ and/or $\vec{B}$ fields, then there is energy. Furthermore, in electromagnetism energy is no longer a property of an object, but some kind of extensive quantity in space. We argue that traditional physics instruction in introductory courses fails to highlight these differences and thus students do not become aware of important traits of the electromagnetic "world view". Analyzing the case of electrostatic energy, we both review critically the standard mechanical discourse and introduce a didactical approach to motivate the electromagnetic way of thinking of energy without the need of an advanced mathematical formalism. The latter is more akin to the practice of present day physics, which relies on lagrangians based on field energy densities.
\end{abstract}

Keywords: Electrostatic energy, electromagnetism, mechanics, physics, epistemology, electromagnetic world view

\section{Introduction}

In the historical development of physics theories it is possible to identify the invention of new concepts, but more often we find a resignification of old ones (see, for instance, Max Jammer's classical books on the evolution of physics concepts). One clear example of this conceptual resignification is the history of energy. In the mechanics framework, energy is closely associated with work and the image it conveys is related to a process that refers to the "past" of a given body or system. For instance, the kinetic energy of a body moving with speed $v$ is equivalent to the work required to bring the body from rest to speed $v$. Similarly, the potential energy of a system is the work necessary to bring the system to a particular configuration, against a conservative force. Furthermore, in the mechanics rationale energy is associated with matter, i.e. one speaks of the energy of an object or a system.

The electromagnetic theory introduced a radically different way to conceive energy. This conceptual shift was explicitly promoted at the end of the 19th century by some of Maxwell's followers such as Poynting, Heaviside and Hertz [1]. The key turning point is traced back to Poynting's seminal paper On the Transfer of Energy in the Electromagnetic Field [2]. Departing from the usual mechanics framework - just as Maxwell did in his Trea-

*Correspondence email address: fkneubil@gmail.com tise [3] - Poynting introduces a new qualitative way to think about energy and applies it in seven examples. In each one of them, Poynting guides our minds to reason about the flow of energy by focusing on the electric and magnetic fields 1 It is possible to say that this work marks a shift in the reasoning about energy from the mechanical language of processes (force, work) to the electromagnetic language of fields in space. Max Jammer highlights some important aspects of this change as follows:

In Poynting's theory of energy flow the transport of energy is no longer confined to conductors. The surrounding medium or empty space is the arena where energy moves. Energy, thus disjoined from mater, raised its ontological status from a mere accident of a mechanical or physics system to the autonomous rank of independent existence, and matter ceased to be the indispensable vehicle for its transport. As a result of this emancipatory change or reification of energy, the idea that only differences in energy are of physical significance had to be abandoned and an absolute magnitude had to be ascribed to energy $([1], p .173)$.

\footnotetext{
${ }^{1}$ Nowadays we write $\vec{S}=\frac{1}{\mu_{0}} \vec{E} \times \vec{B}$ where $\vec{S} \asymp$ is Poynting vector and represents the flux of energy in $W / \mathrm{m}^{2}$.
} 
This new image of the world is one of the most important traits of field theories and conveys a very different mindset when compared with the force and work framework. Despite its importance, the electromagnetic way of conceptualising energy is rarely present in introductory courses. We argue that focusing on the relation between energy and field as soon as possible is essential for providing a better understanding of how electromagnetism, as a field theory, work ${ }^{2}$

This is important to the education of young scientists, because the presence of fields in contemporary physics is overwhelming and theories, such as Quantum Electrodynamics, Quantum Chromodynamics, among others, are expressed in terms of lagrangian densities. We feel that the earlier students are exposed to this way of looking at the world, the better for their formation. In this paper, we choose the electrostatic energy as an example to highlight the differences between the force/work and field approaches and propose a didactical strategy to introduce the latter.

We start by reviewing the traditional derivation of the electrostatic energy of two systems - two point charges and capacitor - using the concepts of force and work, and discuss some of the difficulties of fitting a fully comprehensive mechanical discourse into the time available for lectures ${ }^{3}$. Next, we introduce a didactical approach to motivate the electromagnetic way of thinking of energy without the need of an advanced mathematical formalism. The same expressions for the previous situations are obtained with the electromagnetic framework and the differences in their "world views", as well as the different questions raised by the two approaches, are highlighted. We do not advocate in favour of the exclusion of the usual derivations via mechanics, let alone that the field approach should precede the classical force/work one. Our main goal here is to stress the conceptual gains associated with the electromagnetic framework.

\section{Electrostatic Energy: the Usual Discourse with Force and Work}

\subsection{Point-like charges}

The typical textbook discourse on electromagnetism begins with electrostatics, the part of the theory that deals with charges at rest and, normally, with no mention to reference frames. As their state of rest suppresses the action of time, one has the feeling that one is dealing with an eternal world. This is the scenario for the introduction of the Coulomb field $\vec{E}$, due to a point-like charge $q$, at

\footnotetext{
${ }^{2}$ Of course one can also give mechanics a field formalism, for instance, in the theory of gravitation. However, it is interesting to note that this formulation was proposed later by Heaviside [4], in analogy with the electromagnetic field.

${ }^{3}$ This might shed light on some of the reasons why students struggle with the concept of electric field, as widely shown in the physics education literature (e.g. [5], 6] and 7]).
}

a point $P$ in space, by means of the expression

$$
\vec{E}=\frac{q}{4 \pi \epsilon_{0}} \frac{\vec{r}}{r^{3}}
$$

where $\vec{r}$ is the vector which links the charge to $P$. In order to associate the field with interactions, one invokes a second point-like charge $Q$ and derives the force it suffers owing to the other one, given by

$$
\vec{F}=Q \vec{E}
$$

In this kind of presentation, one normally omits the information that two charges cannot remain at rest by themselves. This qualitative omission is important, for the use of correct equations does not ensure that the approach adopted is logical.

After electric field and force, one introduces the potential energy, usually already discussed in a previous mechanics course. The concept of work of a force $\vec{F}$, along a displacement $d \vec{\ell}$

$$
d W=\vec{F} \cdot d \vec{\ell}
$$

is recalled and combined with eqs. (1) and (2). When work is used in the derivation of the electrostatic potential energy of two point-like charges, displacements of charges along a path between two arbitrary points in space are required. This apparently simple procedure involves an intellectual trick: the displacement required is not based on actual movements but is, rather, an intellectual construction. Even if this may seem too abstract to beginners, one must and does go on, by choosing a path, usually a radial straight line, which is to be made more general later. This allows one to write $d \vec{\ell}=d r \vec{r}$ as being parallel to $\vec{F}$ and the work to bring the charge $Q$ from point $\mathrm{A}\left(\vec{r}_{A}\right)$ to point $\mathrm{B}\left(\vec{r}_{B}\right)$ is given by

$$
\begin{aligned}
W_{A B} & =\int_{A}^{B} \vec{F} \cdot d \vec{\ell}=\int_{r_{A}}^{r_{B}} d r \frac{q Q}{4 \pi \epsilon_{o}} \frac{1}{r^{2}} \\
& =-\frac{q Q}{4 \pi \epsilon_{o}} \frac{1}{r_{B}}+\frac{q Q}{4 \pi \epsilon_{o}} \frac{1}{r_{A}} .
\end{aligned}
$$

Next, one must argue that the work $W_{A B}$ is related to energy, and recalls the living forces theorem, which yields

$$
W_{A B}=\frac{m v_{B}^{2}}{2}-\frac{m v_{A}^{2}}{2},
$$

where $m$ and $v$ are respectively the mass and velocity of the body. Of course, the teacher knows that these symbols refer to properties of a mechanical body. However, charges and bodies are very different things. Moreover, the previously static charges acquire kinetic energy. Students are therefore entitled to see this sudden transformation of static point-like charges into a moving bodies as being both ad hoc and arbitrary.

As the discourse has to go on, results (4) and (5) are used to induce the conclusion that the work of the 
electrostatic force is also related with energy. With this purpose, one writes

$$
\begin{aligned}
W_{A B} & =\frac{m v_{B}^{2}}{2}-\frac{m v_{A}^{2}}{2}=-\frac{q Q}{4 \pi \epsilon_{o}} \frac{1}{r_{B}}+\frac{q Q}{4 \pi \epsilon_{o}} \frac{1}{r_{A}} \\
& \rightarrow \frac{m v_{B}^{2}}{2}+\frac{q Q}{4 \pi \epsilon_{o}} \frac{1}{r_{B}}=\frac{m v_{A}^{2}}{2}+\frac{q Q}{4 \pi \epsilon_{o}} \frac{1}{r_{A}}
\end{aligned}
$$

As the value of the bottom line does not depend on the point considered, one may be tempted to identify it with the total energy of the system and, subsequently, the term proportional to $q Q$ as the potential energy. However, strictly speaking, one should not use results based on the Coulomb field for moving charges. Eq. (6) corresponds then to an approximation, valid for low velocities only, a point not usually discussed in textbooks. Moreover, in mechanics, the potential energy is determined up to an unknown constant $K$, since it is based on the work needed to set the system in a given configuration, departing from another one, chosen arbitrarily as having no potential energy. This point, again, may seem too cumbersome to students.

For the sake of simplicity, one may choose $K=0$ in eq. (6), which amounts to setting the reference level at infinity. Eventually, and in spite of possible gaps in the logical discourse, one reaches the expression for the potential energy of two point-like charges separated by a distance $d$, which is given by

$$
U=\frac{q Q}{4 \pi \epsilon_{0}} \frac{1}{d} .
$$

This result has costs and benefits, that must be assessed. It does look simple, but its physical content still depends on a surrounding conceptual environment. Let us consider the symbol $d$, for instance. Taken at its face value, it represents the distance between the point-like charges. However, it also corresponds to the end-point of a virtual path, needed to bring the charges from a configuration in which they were infinitely separated to another one, where their relative distance is $d$. The point to be stressed here is that the derivation of eq. (7) involves a large number of decisions, which demand some maturity in physics. The problem is not mathematics, since all passages made are simple but, rather, the many verbal provisos which deviate one from the mathematical track. Both the quantity and the apparent artificiality of intermediate explanatory remarks may prevent students from grasping the physics. At the end, one gives the fish, without teaching how to fish. This kind of situation may convey to students a feeling that they are in the midst of a process full of arbitrariness.

\subsection{Capacitors}

The case of capacitors is similar. Owing to their conceptual simplicity, they are important in textbook discussions of potential energy. A pedagogical capacitor can be made by two flat and parallel metal plates of area $S$, separated by a small distance $a$. When the plates are connected to a battery, they get charges $+Q$ and $-Q$, and an electric field $\vec{E}$ appears in the surrounding empty space, both between and outside the plates. As it is difficult to evaluate this field using simple analytic methods, one resorts to the infinite plane approximation, which amounts to a homogeneous field confined just to the region between the plates. Then, Gauss' law yields

$$
|\vec{E}|=\frac{\sigma}{\epsilon_{0}}=\frac{Q}{S \epsilon_{0}} .
$$

In order to evaluate the energy accumulated within the system, the usual procedure, based on mechanical work, consists in making a thought experiment, assuming that there is no initial charge in the capacitor and to carry hypothetical charge elements, from one plate to the other, until the full charge $Q$ is restored. In this way, one gets the electrostatic potential energy of the system, which is given by

$$
U(a)=\frac{1}{2} \frac{Q^{2}}{S \epsilon_{0}} a,
$$

where we have stressed its dependence on the separation $a$ between the its flat plates. The derivation of this result looks simple only if one does not think about it. Here, again, one must resort to virtual displacements with constant velocity and students may want to ask why, now, $U \rightarrow \infty$ when the two plates are set well apart, and not $U \rightarrow 0$, as in the case of two point-like charges. Again, this is not a difficult problem, but there is no short satisfactory answer to it.

This discussion could be extended, but it already suffices to show that the introduction of potential energy in basic undergraduate courses on electromagnetism is not a particularly pleasant one. Courses begin by promoting a world view based on charges and fields, with their ontology of spatial extension, and it may intellectually disappointing for students to be conducted back to mechanical modes of reasoning in the treatment of energy. Especially if this is accompanied by several technical subtleties, which demand lengthy and time consuming explanations.

\section{Restoring the Fields}

The path followed to introduce electrostatic energy only becomes meaningful when looked at a distance, after it is completed. In principle, this is not a problem as, in physics, it is usual that one does research employing results which are just partially understood. People engaged in these activities are quite aware of this, indicating that the same is acceptable in physics teaching, provided that one has enough maturity. The problem occurs when a cumbersome discourse used to introduce a subject is presented as conveying sharp logic. 
In this work, we argue that the world view associated with electrostatic potential energy does have a role to play in education. The widespread notion that the potential energy of a spring is located within itself is quite instructive. However, the treatments introductory textbooks give to potential energy, both gravitational and electrostatic, normally skip the issue of their localization in space, in spite of the close similarities with the case of springs. In undergraduate courses on electromagnetism, the localization of the electrostatic energy can be discussed just after its introduction based on mechanical work. This has the advantage of bringing back the electric field into the physical picture and being easier to understand. In the long run, it also paves the way for going beyond electrostatics, by allowing students to begin to learn the language to be fully explored later, in electrodynamics.

The alternative procedure for treating the electrostatic energy $U$ of a system employs the idea that this energy is spread over space, with a volume density given by

$$
\frac{d U}{d V}=\frac{\epsilon_{0}}{2} \vec{E}^{2},
$$

where $\vec{E}$ is the electric field and $d V$ is the volume element at the point considered. This is the electrostatic version of a quite general result from electrodynamics: if an electric field $\vec{E}$ and a magnetic field $\vec{B}$ exist in a region without charges and currents, the electromagnetic energy density there reads

$$
\frac{d U}{d V}=\frac{\epsilon_{0}}{2} \vec{E}^{2}+\frac{1}{2 \mu_{0}} \vec{B}^{2}
$$

This result can be derived rigorously from Maxwell's equations and the interested reader is directed to traditional electromagnetism textbooks. In electrostatics, the mechanisms which generate the magnetic field $\vec{B}$ are turned off and one recovers eq. (10). The dependence of $d U / d V$ on the square of the fields is, of course, compatible with the fact that it is a scalar quantity, whereas fields are vectors. The importance of these results is that they are local and state that the electromagnetic energy of a system is distributed in space, as the fields are. If there are fields at a point, there is also a certain amount of energy there. This holds for all sorts of situations, ranging from a simple capacitor to interfering electromagnetic waves.

The electrostatic energy density, given by eq.10), is related to the total potential energy of a system by a volume integral, of the form

$$
U=\iiint d V\left[\frac{\epsilon_{0}}{2} \vec{E}^{2}\right],
$$

over the whole volume occupied by the fields. This expression sheds light on the ontological differences between potential energy in mechanical and electromagnetic discourses. In the former, one evaluates a macroscopic global quantity, whereas the latter is more microscopic and therefore, gives rise to a picture of the world which is both more comprehensive and more interesting.

The full electrodynamics treatment of the subject is not suited to basic undergraduate courses. There, it is more convenient to introduce eq. 10 by means of plausibility arguments. This path is followed, for instance, in the textbook Electricity and Magnetism, by Purcell ( 8], p.31-33), who motivates the expression for the energy density by using the case of a charged sphere. Here, instead, we discuss the case of a plane capacitor, as in Ref. 9], which is somewhat simpler.

\subsection{Potential energy of a capacitor}

The energy contained in the capacitor described in the previous section, given by eq. (9), is linear in the interplate separation $a$. If its charge is kept constant and the separation between the plates is increased to a value $b>a$, still small, as in fig, 1, the modulus of the electric field remains the same as that given by eq. (8), and the potential energy becomes

$$
U(b)=\frac{1}{2} \frac{Q^{2}}{S \epsilon_{0}} b>U(a) .
$$

The energy increase $\Delta U=U(b)-U(a)$ can be ascribed to the work done by an external force $\vec{F}_{\text {ext }}$, needed to separate the plates. Considering the upper plate to be positively charged and fixed, the $\vec{F}_{\text {ext }}$ which displaces the negative lower plate must be, at least, equal in modulus to the electric force $\vec{F}_{+}$which attracts it to the other.

This electric force is obtained by considering that each charge in the lower plate feels the electric field $\vec{E}_{+}$due to the upper plate. In the infinite plane approximation, this field is uniform, with an intensity given by

$$
\left|\vec{E}_{+}\right|=\frac{\sigma}{2 \epsilon_{0}},
$$

as illustrated in figure 2(a). All the charges of the lower plate, in figure 2(b), are immersed in the field $\vec{E}_{+}$and

$$
\left|\vec{F}_{+}\right|=Q\left|\vec{E}_{+}\right|=Q \frac{\sigma}{2 \epsilon_{0}}=\frac{Q^{2}}{2 S \epsilon_{0}} .
$$

Thus, the work done by the external agent for separating the plates is

$$
W=\left|\vec{F}_{e x t}\right|(b-a)=\frac{Q^{2}}{2 S \epsilon_{0}}(b-a)=U(b)-U(a) \text {. }
$$

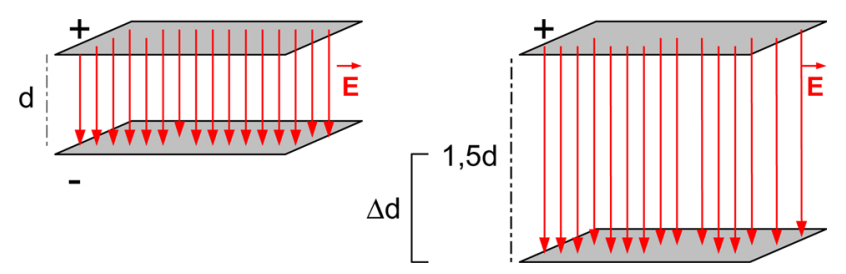

Figure 1: Separation of plates 
As expected, this result shows that the work $W$ accounts for the difference in the potential energies of the system. This looks like an ordinary exercise, but it prepares one to motivate the introduction of eq. 10 .

At this point, it is interesting to ask students to consider the proposition: if the potential energies of the two systems, shown in fig.1, are different, this difference must be directly associated with physical aspects of the system which did change when one moved from one configuration to the other.

In order to guide the ensuing discussion, it is worth distinguishing the features of the system which do not change. Within the infinite plate approximation, these include: (i) the absence of electric field inside the metallic plates; (ii) the way charges are distributed over the inner surfaces of the plates; (iii) the absence of electric field outside the plates; (iv) the direction of the electric field $\vec{E}$ in the region between the plates and (v) the modulus $|\vec{E}|=Q /\left(S \epsilon_{0}\right)$ of electric field in the region between the plates. Concerning the features that do change, one has: (i) the conspicuous increase in the inter-separation distance, from $a$ to $b$, which is geometrical and (ii) the fact that this geometrical change is accompanied by the filling of the volume $S(b-a)$ with electric field.

This last statement relates directly the increase of potential energy with the existence of electric field in space. In order to explore this relationship, we recall that the volume occupied by the field when one passes from one configuration to the other is $\Delta V=S(b-a)$ and, in the case of the plane capacitor, the volume density of potential energy, is given by

$$
\frac{d U}{d V}=\frac{\Delta U}{\Delta V}=\frac{U(b)-U(a)}{S(b-a)}=\frac{1}{2} \frac{Q^{2}}{S^{2} \epsilon_{0}} .
$$

Using eq. (8), we obtain

$$
\frac{d U}{d V}=\frac{\epsilon_{0}}{2} \vec{E}^{2}
$$

which is eq.10). Once this result is reached, it is important to tell students that it is rather general.

In order to fix the idea that electrostatic energy is directly associated to the electric field, one could use

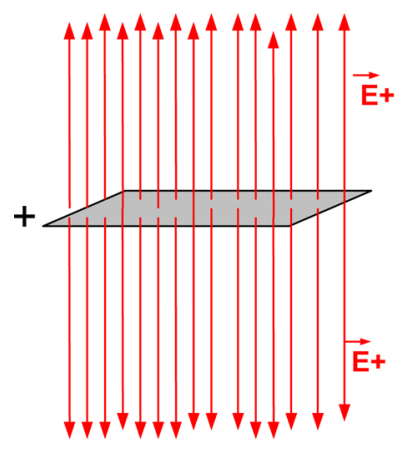

(a)

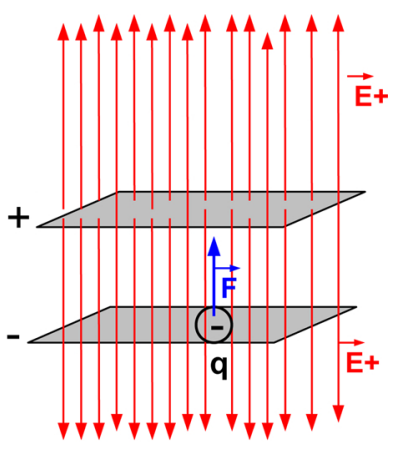

(b)
Figure 2: field and force over a charge eq. (10) to calculate again the energy of the plane capacitor, given by eq. (9). Now, this can be done just by multiplying the energy density by the volume between the plates:

$$
U=\left[\frac{d U}{d V}\right][S a]=\left[\frac{Q^{2}}{2 \epsilon_{0} S^{2}}\right][S a]=\frac{1}{2} \frac{Q^{2}}{S \epsilon_{0}} a,
$$

\subsection{Potential energy of a sphere}

The confidence of students on result 10 can be reinforced by means of other examples. An interesting one concerns the self-energy of a spherical surface of radius $R$, bearing a total charge $Q$. In order to evaluate it by reasoning in terms of force and work, as usually found in textbooks, one assumes the surface to be initially without charge and then begins to bring charge elements $d q$ from infinity. In an intermediate situation, in which the charge of the surface is $0<q<Q$, the total work done by an external force which compensates the electrostatic repulsion reads

$$
d U=\frac{d q q}{4 \pi \epsilon_{0}} \frac{1}{R}
$$

and the total potential energy is

$$
U=\int_{0}^{Q} \frac{d q q}{4 \pi \epsilon_{0}} \frac{1}{R}=\frac{1}{2} \frac{Q^{2}}{4 \pi \epsilon_{0}} \frac{1}{R} .
$$

In the approach based on the energy density, deals just with the full charge $Q$ and writes

$$
\frac{d U}{d V}=\frac{\epsilon_{0}}{2}\left[\frac{Q}{4 \pi \epsilon_{0}} \frac{1}{r^{2}}\right]^{2}
$$

together with

$$
\begin{aligned}
U & =\iiint d V \frac{\epsilon_{0}}{2}\left[\frac{Q}{4 \pi \epsilon_{0}} \frac{1}{r^{2}}\right]^{2} \\
& =\int_{R}^{\infty} 4 \pi r^{2} d r \frac{\epsilon_{0}}{2}\left[\frac{Q}{4 \pi \epsilon_{0}} \frac{1}{r^{2}}\right]^{2} \\
& =\frac{1}{2} \frac{Q^{2}}{4 \pi \epsilon_{0}} \frac{1}{R}
\end{aligned}
$$

This example is interesting because it involves integrations, respectively over $d q$ and $d V$, which convey different ontological contents. The former represents a succession of virtual actions, developed along a kind of logical time, whereas the latter involves a "static" sum over space (from $R$ to $\infty$ !). As these crucial differences may go unnoticed to students, it is important that they are asked to produce their own assessments regarding possible implications of each approach. This may open the way for epistemological discussions, based on concrete instances.

\subsection{Two point-like charges}

Our last example concerns the energy of two point-like charges, already evaluated through the mechanical work 
in eq. (7). Potential energy, whether electrostatic or gravitational, is usually associated with a system and not with charges or bodies. This is so, even if sometimes one finds loose statements concerning the potential energy of an electron in an accelerator or the potential energy of a stone of mass $m$ at Earth's surface as being $m g h$. In the latter case, for example, one knows that the bodyEarth interaction remains tacit in the gravitational field g. Electromagnetism, however, has taught us to move beyond these limitations.

In the case of two point-like charges $q$ and $Q$, located at points $P_{q}=(0,0,-d / 2)$ and $P_{Q}=(0,0,+d / 2)$, the field $\vec{E}$ at the generic point $P$ in space, described in cylindrical coordinates as $P=(\rho \cos \theta, \rho \sin \theta, z)$, is given by

$$
\vec{E}=\vec{E}_{q}+\vec{E}_{Q}
$$

with

$$
\begin{gathered}
\vec{E}_{q}=\frac{q}{4 \pi \epsilon_{0}} \frac{\overrightarrow{r_{q}}}{r_{q}^{3}}, \quad \overrightarrow{r_{q}}=(\rho \cos \theta, \rho \sin \theta, z+d / 2) \\
\vec{E}_{Q}=\frac{Q}{4 \pi \epsilon_{0}} \frac{\overrightarrow{r_{Q}}}{r_{Q}^{3}}, \quad \overrightarrow{r_{Q}}=(\rho \cos \theta, \rho \sin \theta, z-d / 2) .
\end{gathered}
$$

According to eq. 10 , the electrostatic energy density at point $P$ is given by

$$
\begin{aligned}
\frac{d U}{d V} & =\frac{\epsilon_{0}}{2} \vec{E}^{2}=\frac{\epsilon_{0}}{2}\left[\vec{E}_{q}+\vec{E}_{Q}\right]^{2} \\
& =\frac{\epsilon_{0}}{2}\left[\vec{E}_{q}^{2}+\vec{E}_{Q}^{2}+2 \vec{E}_{q} \cdot \vec{E}_{Q}\right] .
\end{aligned}
$$

The interpretation of the three terms in the bottom line of this result is quite interesting and reveals the existence of something new, not foreseen in the mechanical approach. The first term depends only on the charge $q$ and remains always the same, independently of the position, or even the very existence, of the other charge. For this reason, this term is called density of self-energy of charge $q$, and describes the effect this charge produces over itself. The second term is totally analogous and corresponds to the density of self-energy of charge $Q$. In the sequence, we will come back to these contributions.

Before, we discuss the third term, which involves the fields from charges $q$ and $Q$ in a symmetric combination and represents the density of interaction energy. One notes that the fields $\vec{E}_{q}$ and $\vec{E}_{Q}$ appear entangled together and the density of interaction energy is given as

$$
\frac{d U_{i n t}}{d V}=\epsilon_{0} \vec{E}_{q} \cdot \vec{E}_{Q}
$$

This expression cannot be written as a sum of more elementary contributions and represents the spatial distribution of potential energy of the system composed by charges $Q$ and $q$. The potential energy of this system corresponds to the total interaction energy spread over space, given by the integral

$$
U_{i n t}=\iiint d V\left[\frac{d U_{i n t}}{d V}\right]=\iiint d V\left[\epsilon_{0} \vec{E}_{q} \cdot \vec{E}_{Q}\right] .
$$

This integral can be calculated by elementary methods $[9]$, but the calculation is both too long and too involved to be presented here. The important point for us is that the result reads

$$
U_{i n t}=\iiint d V\left[\epsilon_{0} \vec{E}_{q} \cdot \vec{E}_{Q}\right]=\frac{q Q}{4 \pi \epsilon_{0}} \frac{1}{d},
$$

which is identical to eq. (7). One notes, however, that the arbitrary integration constant $K$, present in the mechanical approach, is now absent. This corresponds to an important difference between the "world views" underlying electromagnetism and mechanics, as described in the passage by Jammer quoted in the introduction. The constant $K$ in the force/work approach indicates that this technique allows one to access just potential energy differences whereas, in electromagnetism, one has a direct measure of energy.

We now come back to the self-energy terms, which are not explicitly present in the standard derivation and yield another indication of the power of electromagnetism. Conceptually the self-energy means that a single charge already has energy associated with its field. If this charge is at rest, this energy is purely electrostatic. If it is moving, one should also account for magnetic contributions, as in eq. 111. Considering the case of a single charge $q$ at rest, its full self-energy is given by

$$
U_{\text {self }}=\iiint d V\left[\frac{\epsilon_{0}}{2} \vec{E}_{q}^{2}\right] .
$$

As this energy refers to a particle at rest, its meaning becomes clear only in the framework of the mass-energy relation defined by relativity. Following Okun [10], we write it as $E_{0}=m c^{2}$, where $E_{0}$ is the energy in the rest frame and $m$ is the relativistic invariant mass. The motivations for this form of the mass-energy relations were discussed by Kneubil [11. As the energy $U_{\text {self }}$ is part of $E_{0}$, one concludes that the electrostatic energy of a charged particle contributes to its mass.

Using the explicit form of $\vec{E}_{q}$ in eq. 24, one has

$$
U_{\text {self }}=\int_{R}^{\infty} d r \frac{1}{8 \pi \epsilon_{0}} \frac{1}{r^{2}}=\frac{1}{8 \pi \epsilon_{0}} \frac{1}{R},
$$

where $R$ is the radius of the charge. In the case of pointlike charges, $R \rightarrow 0$ and $U_{\text {self }}$ diverges. The history of this problem is rather rich and a proper discussion is well beyond the scope of this paper. And it still haunts contemporary quantum electrodynamics.

Our purpose here is mainly to stress that the direct association of the electrostatic energy with electric fields can and should be present in introductory undergraduate courses on electromagnetism. It is free from the cumbersome verbal provisos of the traditional force/work 
approach and yields a clear physical picture, by means of eq. 10. Moreover, it gives rise to broader and more powerful results. In association with the magnetic field, it accounts for many other aspects of the nature, which go beyond the potential energy, such as the self-energies of charged particles and the energy of electromagnetic waves. It brings, therefore, the possibility of more profound interpretations about electromagnetic phenomena in nature.

\section{Final Discussion}

We have shown that a standard electromagnetic problem can be solved by means of two alternative approaches, which we suggest to be taught in a close sequence. The didactical relevance of this procedure can be understood by making explicit some epistemological assumptions employed tacitly. The inclusion of elements of epistemology in physics teaching should not be taken as a kind of luxury associated with the wasting of "useful" time in the classroom. Rather, when dressed in actual physics problems, as in the instance discussed here, it becomes a means of bringing to students a mode of dealing with physics which is close to what many scientists do. The close relationship between science and epistemology was stressed by Einstein, in 1949 ([12], p. 684):

The reciprocal relationship of epistemology and science is of noteworthy kind. They are dependent upon each other. Epistemology without contact with science becomes an empty scheme. Science without epistemology is - insofar as it is thinkable at all - primitive and muddled.

The mechanical and electromagnetic approaches to potential energy involve rather different world views. A world view, in Dewitt's definition, consists of an interconnected set of symbolic elements which allows one to give meaning to an entity or phenomenon that one intends to know 13. As a consequence, the power of isolated ideas to produce meanings for the natural world is rather limited. Thus, the character of net or conceptual structure which underlies a world view forces one to consider that the process of its construction involves a weaving of meanings which transcends the simple relationship between an entity and its measurement.

Our suggestion that both approaches to the electrostatic energy should be presented in sequence is intended not only to enrich the acquaintance with the specific subject, but also to explore the possibilities open for reflection about science. This is aligned with Thomas Kuhn's [14] argument that scientific formation occurs dominantly through exemplary problems, which are not just means of applying knowledge learned somehow previously. The construction of the solution to a problem and its implementation amount to the very elaboration of the scientific thought.
Mechanics, with the epistemological project established by Newton and followers, considers interactions between bodies. However, the very nature of these interactions is not discussed and phenomena are reduced to individual actions. This leads to the construction of a world view in which interactions are mere requirements needed to justify the existence of forces, these being the actual cause of phenomena, such as changes in the state of motion of bodies. This version acquires strength with the concept of work which relies on actions of forces on bodies. Accordingly, mechanics emphasizes the parts of a system, which may be scrutinized as individual units.

The world view based on electromagnetism, the framework proposed at the end of the nineteenth century, involves another epistemology. In this case, the fundamental interest is the very nature of interactions, which become the protagonists. Charges are assumed to be the sources of fields, which acquire an autonomous "reality" and become the conveyors of physical qualities. The dealing with fields requires a perception of totalities because, in essence, one looks for a topology in space whereby the fields ascribe vectors to each of its points.

The very concept of energy ends up being resignified within this new framework. The systemic and "holistic" character of energy derives from the mode of thought embodied into electromagnetism, which does not reduce a totality to its parts. Going back to the instances discussed in the previous section, the procedure for obtaining the energy by means of the volume integral of a density based on the field amounts to locate this energy all over space. However, the most interesting feature suggested by this approach consists in realizing that energy totalities involve terms referring both to individual charges only (self-energy) and others which encompass the whole system necessarily (interaction energy). Here, in summary, the whole transcends its parts.

The instances discussed in this paper show that the different solutions convey their own world views. Both scientific and educational practices do not require necessarily that physics should amount to a world view and legitimate work can be produced renouncing to it. However, if one sticks to the so called "cultural value" of sciences, it becomes difficult to dismiss epistemological discussions which can highlight the world view underlying a theory. Even within this more restricted perspective, the specific situation tackled in this work, the early introduction of students to the picture of potential energy based on fields is welcome, since it paves the way both to more advanced courses on electromagnetism and to the practice of contemporary theory which relies largely on lagrangian densities. On the other hand, when one looks at concepts from a more refined perspective, theories must be suitably taught in classroom. Therefore, the understanding of electromagnetic theory can only be complete if the solutions to its exemplary problems could display its world view in full. 


\section{Acknowledgements}

F.B.K. thanks the financial support by CAPES (BEX 0816/15-9) and M.R.R. thanks the hospitality of the Department of Science Education of the University of Copenhagen and the financial support by FAPESP (Brazilian Agencies).

\section{References}

[1] M. Jammer,. Concepts of Mass in Classical and Modern Physics (Harvard University Press, New York, 1961).

[2] J.H. Poynting, Phil. Trans. R. Soc. Lond. 175, 343 (1884).

[3] J.C. Maxwell, A Treatise on Electricity and Magnetism (Cambridge University Press, Cambridge, 1873), available from http://dx.doi.org/10.1017/ CB09780511709340 accessed May 26, 2016.

[4] O. Heaviside, The Electrician 31, 281 (1893).

[5] L. Viennot and S. Rainson, International Journal of Science Education 14, 475 (1992).

[6] I. Galili, International Journal of Science Education, 17, 371 (1995).

[7] C. Furió and J. Guisasola, Science Education 82, 511 (1998).

[8] E. Purcell and D. Morin, Electricity and Magnetism (Cambridge University Press, Cambridge, 2013), 3rd ed.

[9] M.J. Bechara, J.L.M. Duarte, M.R. Robilotta and S. Salém, Física 4, Lecture Notes University of São Paulo, São Paulo, 2014).

[10] L.B. Okun, Physics Today 42, 31 (1989).

[11] F.B. Kneubil, The Epistemological Path of Knowledge and the Mass-Energy Equivalence. Ph.D Thesis (text in Portuguese), University of São Paulo, 2014.

[12] P.A. Schilpp (ed.) Albert Einstein: Philosopher-Scientist (The Library of Living Philosophers, Evanston, 1949).

[13] R. DeWitt, Worldviews: An Introduction to the History and Philosophy of Science (Wiley-Blackwell, Oxford, 2003).

[14] T.S. Kuhn, The Structure of Scientific Revolutions (University of Chicago Press, Chicago, 1962). 